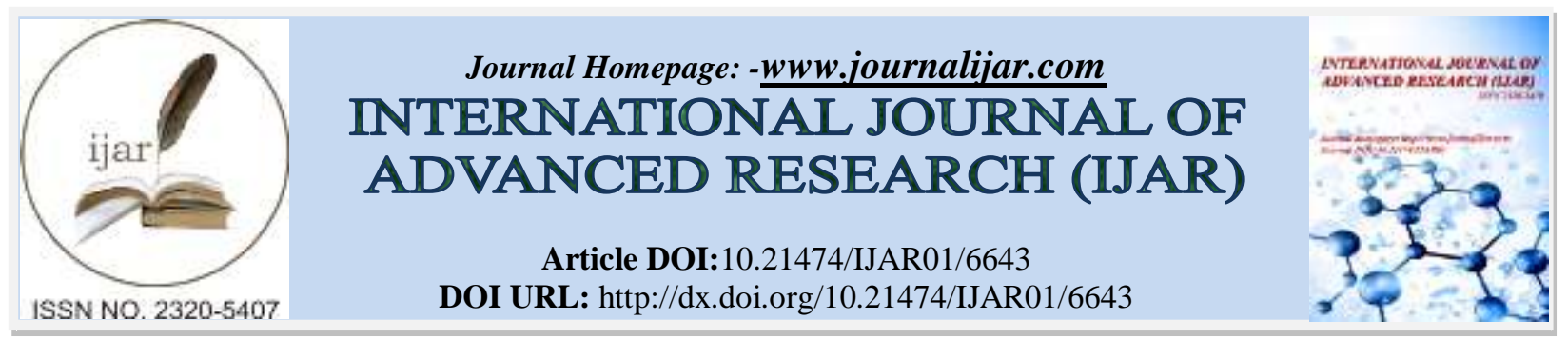

RESEARCH ARTICLE

\title{
PROFIL MORPHO-CONSTITUTIONNEL DE LA LITHIASE URINAIRE EN MAURITANIE MORPHO-CONSTITUTIONAL PROFILE OF UROLITHIASIS IN MAURITANIA.
}

\section{T I. Boudhaye ${ }^{1,2}$, A. Abdelmalick ${ }^{2,3}$, M.Sidbrahim ${ }^{1,2}$, C. Jdoud ${ }^{2,4}$ and SM. Ghber ${ }^{2,5}$.}

1. Service D'urologie, Hôpital Militaire De Nouakchott.

2. Faculté de médecine de Nouakchott.

3. Service de biologie, Hôpital Militaire de Nouakchott.

4. Service d'urologie, Centre Hospitalier National.

5. Service de biologie, Centre Hospitalier National.

\section{Manuscript Info}

\section{Manuscript History}

Received: 02 January 2018

Final Accepted: 04 February 2018

Published: March 2018

Keywords:-

Morpho-constitutional analysis;

urolithiasis ; Mauritania.

\section{Abstract}

Context: The incidence and prevalence of urolithiasis is increasing in the world, including regions that have historically had low urolithiasis rate. This is explained by the trend of global warming, dietary changes and obesity.

Objective: The aim of this study was to describe to identify the chemical composition of urinary stone and to identify the main risk factors.

Materials and Methods: 40 patients with urinary stones extracted by intervention or spontaneously expelled, was collected over a period of 20 months with regular follow-up of all patients.

Results:For 35\% of our patients the age range was $40-59$ years, with an equal percentage for the other age range, the male was affected in $90 \%$, the origin corresponded in $40 \%$ to a semi- wet followed by wetland $(32.5 \%)$. Low water intake (less than one liter per day) was observed in $43.6 \%$, an excessive consumption of tea (greater than five times a day) was observed in $43.6 \%$. Oxalocalcic calculations were predominant (67.5\%) with rates of $47.5 \%$ for whewellite and $20 \%$ for weddellite, followed by urine calculations in $20 \%$ and phosphocalcic in $12.5 \%$.

Conclusion:Urolithiasis affects all age groups, especially young adults. The hot climate and the excessive consumption of lithogeny foods would seem to be the main lithogeny risk factors in our country. For chemical nature, it resembles that observed in neighboring countries, and tends now to follow those of industrialized countries.

Copy Right, IJAR, 2018,. All rights reserved.

\section{Introduction:-}

La lithiase urinaire constitue un problème majeur de santé publique, l'incidence et la prévalence des lithiases urinaires sont en augmentation dans le monde [1].

La Mauritanie n'échapperait pas à cette tendance malgré l'absence de données épidémiologiques fiables. 
L'analyse physico-chimique des calculs urinaires apporte des informations qui peuvent contribuer efficacement à la compréhension des mécanismes impliqués dans leur formation. Elle devrait donc être le premier temps de l'exploration étiologique.

L'identification des causes de la lithiase permet de lui opposer des mesures thérapeutiques ou diététiques efficaces conduisant à la réduction ou à l'arrêt des récidives [2].

Dans notre pays, aucune étude ne s'est intéressée à la composition physico-chimique des calculs urinaires. La présente étude a pour but de cerner le profil morpho-constitutionnel de la lithiase urinaire en Mauritanie.

\section{Patients Et Méthodes:-}

Nous avons colligé 40 patients ayant présenté des calculs urinaires dont 39 extraits par voie chirurgicale et un expulsé spontanément.

Une fiche d'enquête a été utilisée, elle mentionnait l'âge, le sexe, l'origine, le motif de consultation, les antécédents et les habitudes alimentaires et les résultats de l'analyse morpho-constitutionnelle du calcul.

Les patients inclus étaient des adultes dont les calculs urinaires étaient expulsés chirurgicalement ou spontanément, ils disposaient tous de l'analyse morpho constitutionnelle du calcul, réalisée par spectrophotométrie infrarouge.

Il s'agissait d'une étude observationnelle descriptive à visée analytique avec collecte de données prospectives, portant sur quarante cas, sur une période de dix-huit mois allant du premier aout 2015 au vingt-huit février 2017, au sein du service d'urologie à l'Hôpital Militaire de Nouakchott.

La saisie de données ainsi que l'analyse statistique ont été effectuées sur le logiciel de traitement statistique (SPSS version 20.0), la saisie bibliographique a été faite à partir du logiciel de gestion bibliographique (EndNOTE X7).

Après vérification et contrôle des données, les techniques statistiques suivantes ont été appliquées :

-tri à plat pour décrire les différentes variables chacune à part.

-croisement des variables pour mesurer la corrélation entre les variables théoriquement liées. Dans ce cadre, le test de chi-deux et le test exact de Fischer ont été utilisés pour les variables qualitatives.

\section{Résultats:-}

\section{Caractéristiques Démographiques:}

La répartition des lithiases selon l'âge, la profession et le sexe était rapporté dans le Tableau I ; le sexe ratio (H/F) était de 12,3, la moyenne d'âge de 47,2 ans.

L'origine démographique était classée selon le gradient de pluviométrie en trois zones, aride, humide et semi humide, les malades étaient majoritairement de la zone semi humide (37,5\%) suivi de la zone humide (35\%) (Fig.1).

\section{Motifs de Consultations et les Antécédents:}

La colique néphrétique était le principal motif de consultation, elle était constatée chez 37,5\% des patients, suivie de la dysurie chez 35\%, la notion d'un traitement médical expulsif était trouvée dans 7,5\%, une notion de lithiase familiale a été observée chez 5\%(voir Tableau. II).

\section{Habitudes Alimentaires et Mode de vie:}

Les habitudes alimentaires étaient utiles à connaitre afin de mettre en exergue les aliments lithogènes les plus utilisés dans notre population comme le thé, la viande rouge et les produits laitiers(voirTableau.III), $52.5 \%$ des patients étaient des tabagiques et seulement $20 \%$ exerçaient une activité physique régulière.

\section{Nature physico-chimique des Calculs:}

L'analyse spectrophotométrique infrarouge a montré des calculs majoritairement calciques (80\%) de type oxalocalcique dans $67,5 \%$ (Tableau.IV), la prévalence de ces différentes natures phyico-chimiques était représentée selon l'âge et le siège des calculs (Fig.2-3). 


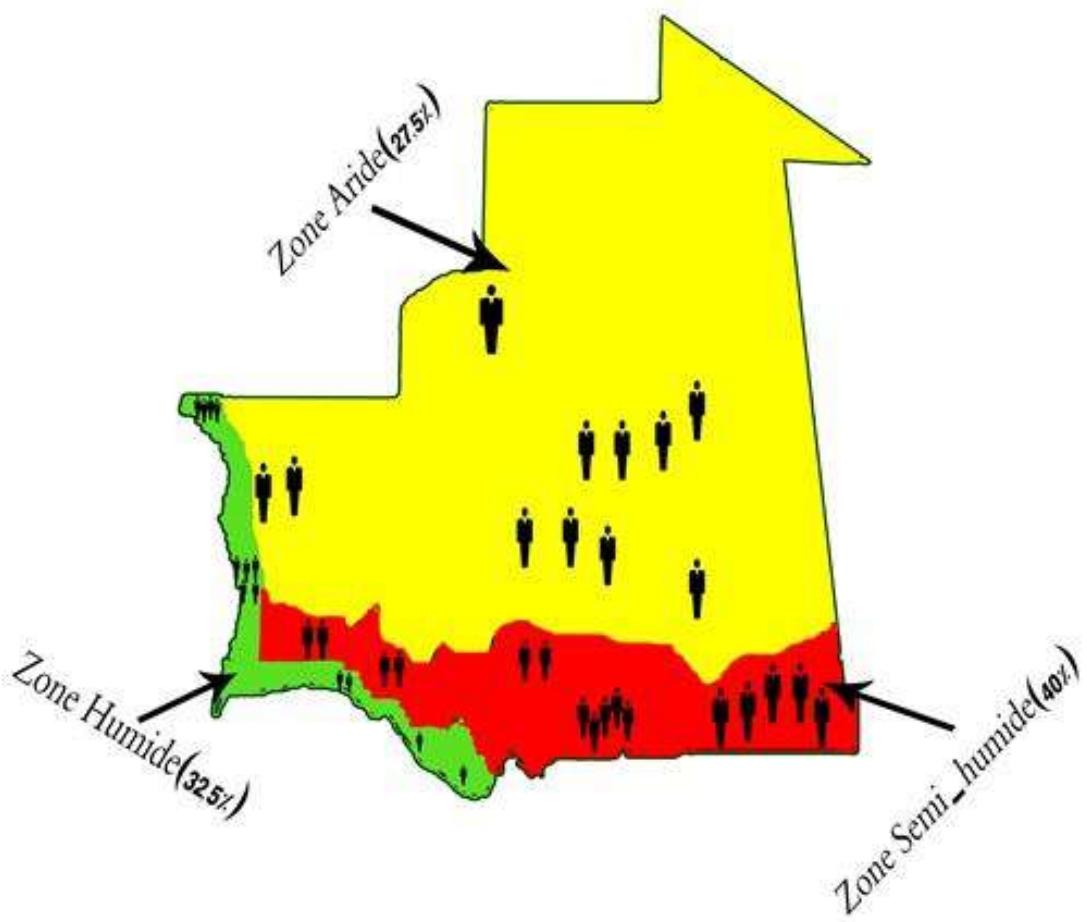

Figure 1:- L'origine géographique selon le gradient pluviométrique Geogrphic origin according to pluviometry gradient.

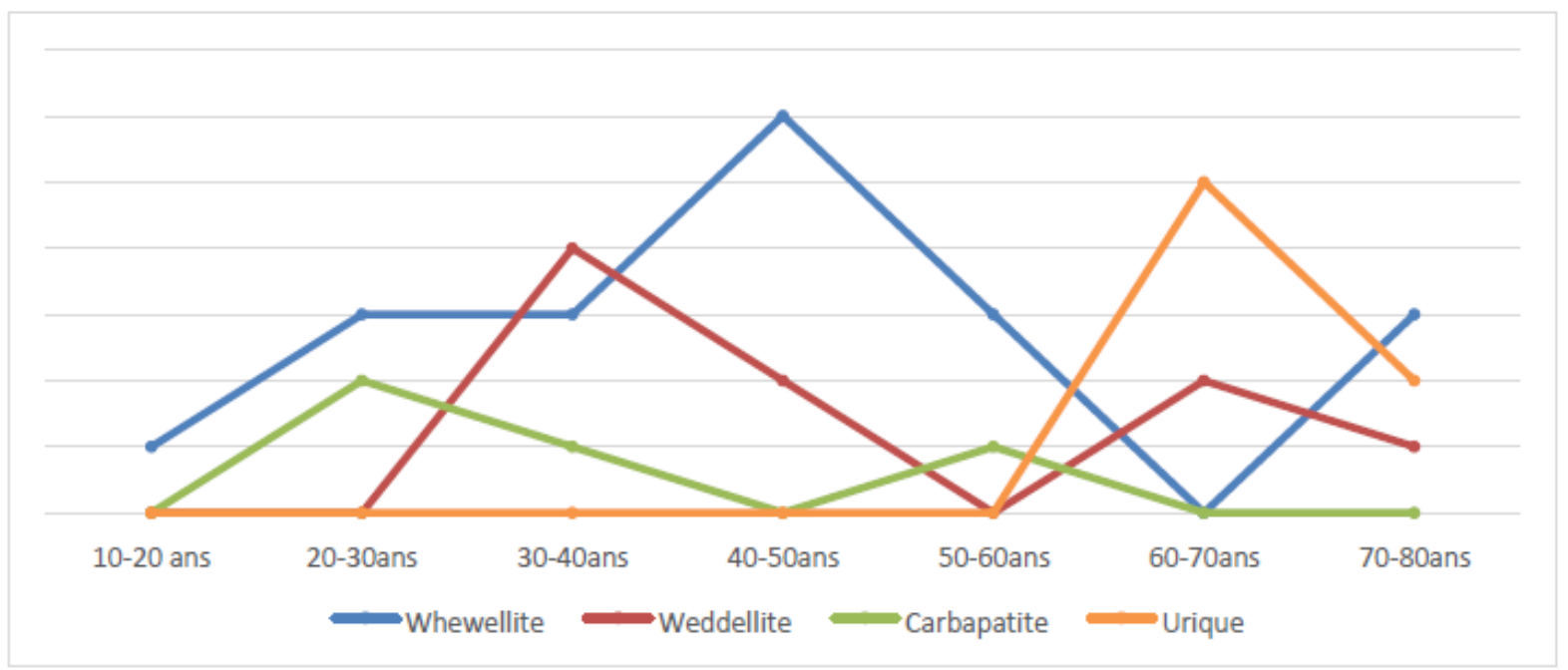

Figure 2:- L'évolution de la prévalence morpho-constitutionnelle des calculs selon l'âge Prevalence to urinary stones according to age. 


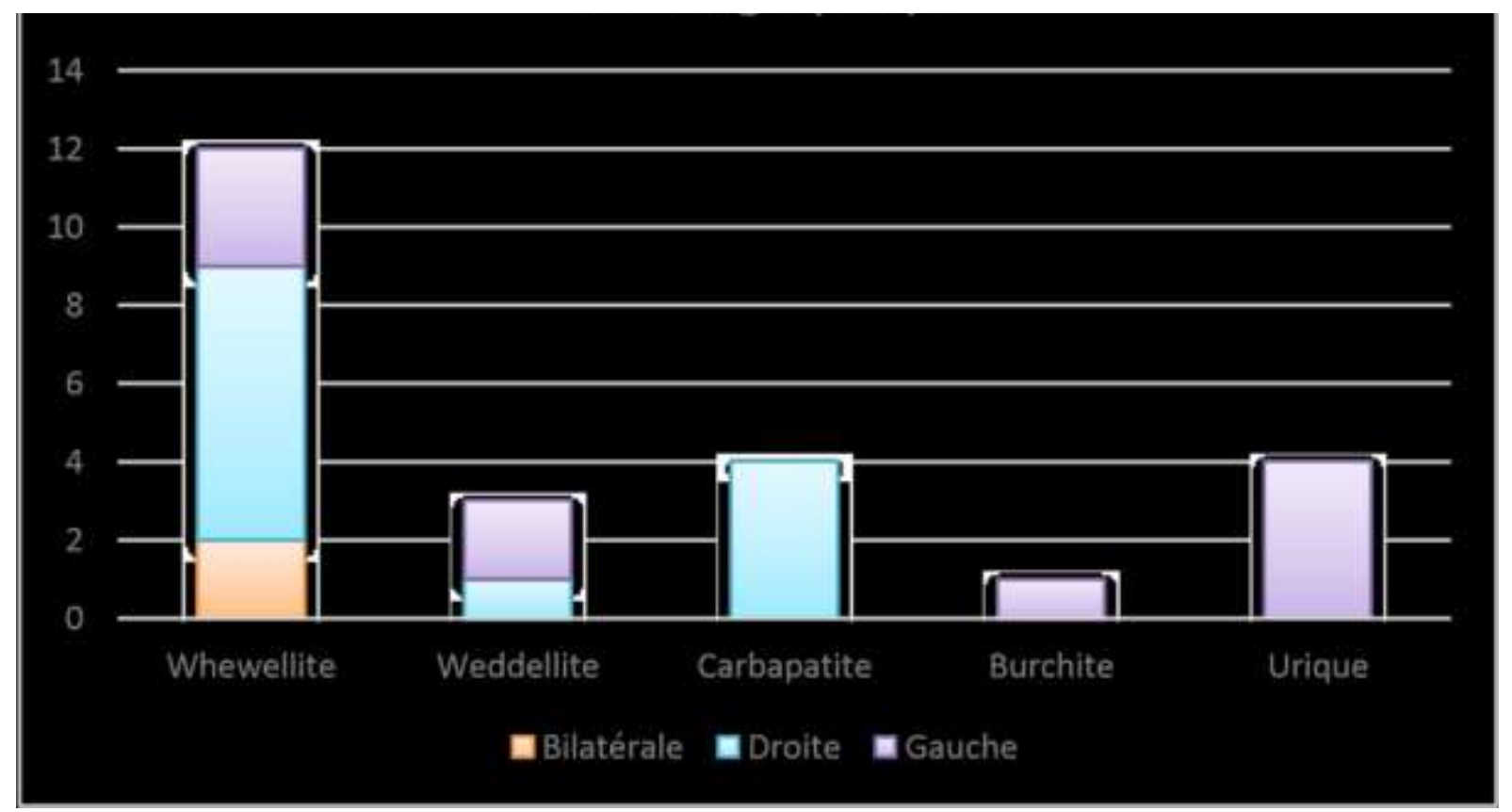

Figure 3:- Siège des calculs selon leurs natures chimiques Location of stones according to chemical analysis

Tableau I:- Répartition selon l'âge le sexe et la profession Distrubition of patients acoording to gender, age and profession

\begin{tabular}{|lcc|}
\hline & Effectif & Pourcentage \\
\hline Age & & $32,5 \%$ \\
$20-39$ ans & 13 & $35 \%$ \\
$40-59$ & 14 & $32,5 \%$ \\
$\geq 60$ & 13 & $90 \%$ \\
Sexe & & $10 \%$ \\
Homme & 36 & \\
Femme & 04 & $55 \%$ \\
Profession & 22 & $35 \%$ \\
Militaire & 18 & $36,8 \%$ \\
Civile & 07 & $21,1 \%$ \\
Fonctionnaires & 04 & $15,8 \%$ \\
Commerçants & 03 & $26,3 \%$ \\
Agriculteurs & 05 & \\
Autres & & \\
\hline
\end{tabular}

Tableau. II:- La répartition selon le motif de consultation Distrubition of patients acoording to consultation reason SBAU: symptômes du bas appareil urinaire

SHAU: symptômes du haut appareil urinaire

\begin{tabular}{|lcc|}
\hline & Effectif & Pourcentage \\
\hline SHAU & 13 & $32,5 \%$ \\
Colique néphrétique & 15 & $35 \%$ \\
Lombalgie & 09 & $22,5 \%$ \\
SBAU & 15 & $37,5 \%$ \\
Dysurie & 14 & $32,5 \%$ \\
Hématurie & 09 & $20 \%$ \\
Brulure mictionnelle & 08 & $22,5 \%$ \\
Pollakiurie & 05 & $12,5 \%$ \\
Douleur pelvienne & 01 & $02,5 \%$ \\
SBAU+SBAU & 07 & $15 \%$ \\
\hline
\end{tabular}




\begin{tabular}{|ccc|}
\hline Complications & 04 & $10 \%$ \\
Insuffisance rénale & 02 & $05 \%$ \\
Pyélonéphrite & 01 & $02,5 \%$ \\
Rétention d'urine & 02 & $05 \%$ \\
Découverte fortuite & 01 & $2,5 \%$ \\
\hline
\end{tabular}

Tableau. III:- La Répartition d'aliments lithogènes Distrubition of foods causing urolithiasis

\begin{tabular}{|c|c|c|}
\hline & ECTIF & POURCENTAGE \\
\hline \multicolumn{3}{|l|}{ Thé par jour } \\
\hline Aucune & 03 & $7,7 \%$ \\
\hline Une fois & 06 & $15,4 \%$ \\
\hline 2 à 3 fois & 13 & $33 \%$ \\
\hline Supérieur à 3 fois & 17 & $43,6 \%$ \\
\hline \multicolumn{3}{|c|}{ Viande rouge par semaine } \\
\hline Aucune & 02 & $05,1 \%$ \\
\hline Inférieur à 3 fois & 11 & $28,2 \%$ \\
\hline 3 à 5 fois & 08 & $20,5 \%$ \\
\hline Supérieur à 5 fois & 18 & $46,2 \%$ \\
\hline \multicolumn{3}{|l|}{ Boisson par jour } \\
\hline Inférieur à $1 \mathrm{~L}$ & 17 & $43,6 \%$ \\
\hline Entre 1 à 2 L & 21 & $53,8 \%$ \\
\hline Supérieur à 2 L & 01 & $02,6 \%$ \\
\hline \multicolumn{3}{|c|}{ Quantité de lait par jour } \\
\hline Inférieur à $0,5 \mathrm{~L}$ & 21 & $53,8 \%$ \\
\hline Entre 0,5 à $1 \mathrm{~L}$ & 16 & $41 \%$ \\
\hline Supérieur à $1 \mathrm{~L}$ & 02 & $05,1 \%$ \\
\hline \multicolumn{3}{|c|}{ Nombre portion légume/fruit par semaine } \\
\hline Aucune & 01 & $02,6 \%$ \\
\hline Mensuelle & 02 & $05,1 \%$ \\
\hline Inférieur à 3 fois & 22 & $56,2 \%$ \\
\hline Entre 3 et 5 fois & 10 & 25,6 \\
\hline Supérieur à 5 fois & 04 & 10,3 \\
\hline
\end{tabular}

Tableau IV: La prévalence des différentes natures physico-chimiques Prevalence of different physico-chemical kinds.

\begin{tabular}{|ccc|}
\hline & Effectif & Pourcentage \\
\hline Calcique & 32 & $80 \%$ \\
Oxalocalcique & 27 & $67,5 \%$ \\
.whewellite & 19 & $47,5 \%$ \\
.weddellite & 08 & $20 \%$ \\
Phosphocalcique & 05 & $12,5 \%$ \\
.carbapatite & 04 & $10 \%$ \\
.brushite & 01 & $2,5 \%$ \\
Uratique & 08 & $20 \%$ \\
\hline
\end{tabular}

\section{Discussion:-}

Cette étude porte sur 40 patients, ayant présenté des calculs de l'arbre urinaire.Elle représente la première étude qui s'intéresse au profil morpho-constitutionnel de la lithiase urinaire en Mauritanie.

\section{Caractéristiques Démographiques:}

La tranche d'âge la plus touchée était entre 40-59 ans avec une moyenne d'âge de $47 \pm 16,2$ ans, ces valeurs sont semblables à ceux observés dans la population tunisienne [3] et américaine [4] mais différentes de celles observées

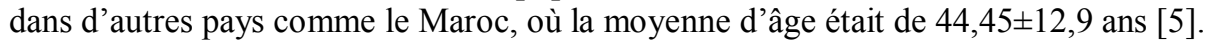


L'étude montre une prédominance de la lithiase chez les hommes avec un sexe ratio $\mathrm{H} / \mathrm{F}$ de 12,3 .Ce résultat est plus élevé par rapport à celui retrouvé au Maroc $(2,1)$ [2], au Burkina-Faso $(1,91)$ [6] et même en Espagne $(1,26)[7]$ mais plus proche de celui rapporté chez les militaires français qui est de 19,3[8], la population était dans 55\% des militaires qui sont en majorité masculins ce qui expliquerait ce ratio.

L'origine semi-humide était majoritaire (40\%), ce résultat est comparable à celui du Burkina-Faso : 93\% [2-6], cela pourrait être dû à la densité élevée dans cette zone [9] mais aussi la chaleur et l'humidité, qui pourraient être des facteurs incriminés selon Bailly, Lee et Oussama [10, 11,12].

\section{Motifs de Consultations et Antécédents:-}

Les symptômes du bas appareil urinaire(SBAU) sont légèrement majoritaires $(37,5 \%)$ par rapport à ceux du haut appareil (32,5\%), la colique néphrétique demeure le maitre symptôme (35\%), comme en Espagne $(52,5 \%)$ et au Burkina Faso (32\%) [6,7].

La maladie de cachi riccie qui est une maladie héréditaire familiale correspondant à la dilatation précalicielle des tubes rénaux, est retrouvée dans 12 à $20 \%$ des lithiases rénales radio-opaques de type oxalocalcique et fait le lit d'infection urinaire [13], elle a été constatée chez un de nos patient.

La présence de lithiase familiale était retrouvée chez $5 \%$ des malades, elle augmente de 2,5 fois le risque de la maladie [14].

Les complications sont présentes dans $30 \%$ des cas, avec une prédominance de l'hydronéphrose : $37,5 \%$, la deuxième complication est l'insuffisance rénale aigue qui était présente dans $5 \%$.

Habitudes Alimentaires et Mode de vie:-

Dans notre échantillon, nous avons constaté

- Un apport excessif des aliments lithogènes: le thé dans $43,6 \%$, la viande rouge dans $46,2 \%$, le lait dans $41 \%$

- $\quad$ un faible apport hydrique : inférieur à $1 \mathrm{~L}$ était observé dans $43,6 \%$

- $\quad$ une faible activité physique était constatée dans $80 \%$.

Ces habitudes expliqueraient la tendance de nos malades à l'hyperoxaliurie et l'hypercalciurie $[4,15,16]$.

L'utilisation excessive des aliments riche en oxalates (thé, chou, cacao,tomate....) a été retrouvée chez $49 \%$ des patients maliens selon M. Yacouba [17].

\section{Analyse physico-chimique des calculs urinaires:-}

\section{Prévalence de différentes Natures physicochimiques:}

L'étude des grandes séries $[18,5,6,35]$ a montré une nette prédominance des calculs oxalocalciques de type whewellite ce qui a été retrouvé dans notre série avec un pourcentage de $47,5 \%$, suivi de calculs oxalocalciques de type weddellite dans $20 \%$, uratique dans $20 \%$ puis le phosphocalciques dans $12,5 \%$.

La prévalence de l'oxalocalcique, était inférieure à celle obtenue au Maroc et en France [20].

Cette tendance de la nature chimique des calculs dans notre pays est identique à celle observée dans les pays en voie de développements d'après les constats de Daudon [21].

L'utilisation excessive de thé chez nos malades entrainerait une hyperoxalurie [22,23], l'hypercalciurie peut être expliquée par les habitudes hygiéno-diététiques de nos malades, notamment la consommation excessive des produits laitiers et la sédentarité.

\section{Influence de l'âge sur la Nature physico-chimique:-}

La proportion des calculs de type whewellite augmente avec l'âge jusqu'à la tranche d'âge 40-50 ans et diminue pour la tranche 60-70 ans puis elle remonte, cette chute peut être expliquée par la taille limitée de cette tranche.

Les calculs phosphocalciques ont une faible incidence, et diminuent avec l'âge. Pour le calcul de type weddellite, il n'apparait à l'âge jeune que dans $20 \%$, ce pourcentage est plus faible que celui constaté dans les pays du Maghreb 
[5,24]. Les calculs uriques apparaissent plus tardivement vers 50-60 ans, cela était observé par EL Fellah au Maroc $[4,25]$.

Bien que nous ne disposions pas de toutes les données métaboliques ni des caractéristiques anthropométriques des patients, il est vraisemblable que ces calculs uratiques soient favorisés par des modifications métaboliques liées au vieillissement et/ou à un syndrome de résistance à l'insuline, entraînant un défaut d'ammoniogenèse rénale et une baisse du $\mathrm{pH}$ urinaire [26].

Un autre facteur favorisant la formation des calculs dans ce contexte métabolique est la stase urinaire induite par une hypertrophie de la prostate. Ces observations doivent nous inciter à rechercher un syndrome métabolique chez tout patient présentant une lithiase urique, a fortiori en présence d'une hypertension artérielle et/ou d'un surpoids.

\section{Siège des Calculs selon la Nature Chimique:-}

Le siège des calculs selon la nature chimique a été différemment expliqué selon les auteurs, pour Daudon et Djelloul[18,24] les calculs sont généralement latéralisés à gauche, Cependant, l'étude de Hesse et al[27] réalisée en Allemagne et portant sur 10000 calculs, n'a révélé aucune différence significative quant à la latéralisation des calculs.

Shekarriz et al ont attiré l'attention sur le lien pouvant exister entre le siège des calculs et le côté sur lequel les patients ont pris l'habitude de dormir [28], dans notre série la latéralisation a été significative du côté droit avec un p-value $<0,05$.

Le composant majoritaire whewellite n'a pas de latéralisation préférentielle pour Daudon [18].

Par contre le phosphocalcique est latéralisé souvent du côté droit, à l'ouest Algérie [24], cette latéralisation est comparable aux données de notre série.

Le weddellite est faiblement latéralisé à gauche, ce constat a été rapporté dans une série de 4860 calculs en France [18]

Dans la série de Djelloul [24], dans deux tiers des cas et pour les 2 sexes la latéralité de l'acide urique etait gauche ; cela est similaire à notre série ou la totalité de ce type de calcul était située du même côté.

\section{Conclusion:-}

La lithiase urinaire est une pathologie qui touche toutes les tranches d'âge de la population avec une légère prédominance de l'adulte jeune.

Du point de vue Morpho-Constitutionnel, la lithiase de nature oxalocalcique était prédominante surtout chez l'adulte jeune, chez le sujet âgé l'apparition du calcul de nature uratique était quasi-exponentielle, ce fait pourrait être lié à l'obstruction des voies urinaires mais aussi aux modifications métaboliques liées au vieillissement et/ou à un syndrome de résistance à l'insuline, entraînant un défaut d'ammoniogenèse rénale et une baisse du pH urinaire.

Ces données concordent avec celles observées dans les pays voisins avec une tendance à suivre celles des pays industrialisés.

Cette étude bien que pionnière, était limitée dans le temps, elle n'a concerné qu'un échantillon de petite taille. Une autre étude de plus grande cohorte serait recommandée pour avoir une idée plus objective sur l'ampleur de cette pathologie dans notre pays. 


\section{Bibliographie:-}

1. OA Raheem, YS Khandwala ,KR Ghani et al. Burden of Urolithiasis: Trends inPrevalence, Treatments, and Costs. European urology focus. 2017;31:18-26.

2. 2.A.Oussama ,M.Daudon et al. Analyse des calculs urinaires de l'adulte dans le Moyen Atlas marocain par spectrophotométrie infrarouge. Prog Urol. 2000;10(3):404-10.

3. A. Alayaa, I. Hellaraa, M. Belgithb et al. Étude de la composition des calculs urinaires en fonction de l'âge dans la population du centre tunisien. Prog.Urol.2012;22:938-44.

4. M.Daudon,O.Traxer.Lithiase urinaire. Medicale sciences publication.

5. Lavoisier, Paris.2012, 170.

6. F.Laziri . Facteurs impliqués dans l'épidémiologie des calculs urinaires marocains.J Maroc Urol. 2010;1(19):9.

7. FA.Kaboré,T.Kambou,B.Zango et al. Épidémiologie d'une cohorte de 450 lithiases urinaires au CHU Yalgado Ouédraogo de Ouagadougou (Burkina Faso). Prog Urol.2013;23(12):971-6.

8. FM.Alapont Pérez ,J.Gálvez Calderón ,J.Varea Herrero et al. Epidemiología de la litiasis urinaria. Actas Urológicas Españolas. 2001;25(5):341-9.

9. H.Abdourahman,A.De Chaumont et al.Epidemiology of urinary stones in the French military during the operation Serval. Prog.Urol. 2014;24(12):764-70.

10. Ao.soule. profil forragier de la Mauritanie. Foot and Agriculture Organization of the United Nations. 2013, 4:102-3.

11. http://www.environnement.gov.mr/fr/index.php/climat-de-lamauritanie

12. 11.V.Bailly,I.Le Ray ,N.Bardonnaud et al. Meteo-U-rology: climate impact on urological emergencies. Prog.Urol. 2014;24(9):535-9.

13. S.Lee ,MS.Kim,JH.Kim et al. Daily Mean Temperature Affects Urolithiasis Presentation in Seoul: a Timeseries Analysis. Journal of Korean medical science.2016;31(5):750-6.

14. F.Bandaly ,J.Rabearivelo, A.Sayegh. La maladie de Cacchi et Ricci : une affection favorisant la lithiase rénale et l'infection urinaire. Journal Européen des Urgences et de Réanimation. 2014;26(1):55-7.

15. 14 .GC.Curhan. Epidemiology of stone disease. The Urologic clinics of North America.2007;34(3):287-93.

16. O.Traxer. Urolithiasis: metabolic evaluation and prevention. Prog.Urol.2012;22(14):876-80.

17. KL.Penniston,SY.Nakada. Diet and alternative therapies in the management of stone disease. The Urologic clinics of North America. 2013;40(1):31-46.

18. MN Traore . Etude des Lithiases de L'appareil Urinaire dans Le Service D’urologie du CHU du point «G» : à propos de 100 CAS: Faculté de Medcine et d'odontostomatologie,Bamako,2011 - 2012,36,59P.

19. M.Daudon ,O.Traxer,E.Lechevallier et al. Epidemiology of urolithiasis.Prog.Urol. 2008;18(12):802-14.

20. V.Castiglione, F.Jouret ,O. Bruyere et al.Epidemiology of urolithiasis in Belgium on the basis of a morphoconstitutional classification. Nephrologie \& therapeutique. 2015;11(1):42-9.

21. R.El Habbani,A.Chaqroune,T.Sqalli Houssaini et al. Epidemiological study on urinary stones in the region of Fez and the risk of recurrence. Prog.Urol.2016;26(5):287-94.

22. M.Daudon, SC.Francisco ,LS.Sônia et al. Composition des calculs observés aujourd'hui dans les pays non industrialisés. Progr Urol. 2004;14:1151-61.

23. .V.Arumuham,J.Bycroft.The management of urolithiasis. Surgery (Oxford).2016;34(7):352-60.

24. O.Traxer,E.Lechevallier,C.Saussine. Diététique et lithiase rénale: le rôle de l'urologue. Prog.Urol. 2008;18(12):857-62.

25. Z.Djelloul,A. Bedjaoui,Z.Kaid-Omar et al. Lithiase urinaire dans l'Ouest algerien: Etude de la composition de 1354 calculs urinaires en relation avec leur localisation anatomique, l'âge et le sexe des patients. Progrès en urologie. 2006;16(3):328.

26. H.El Fellah. Aspects épidémiologiques de la lithiase urique: influence de l'âge, du sexe et du diabète. J Maroc Urol. 2009;1(15):15-21.

27. F.Domingos F, Serra A. Metabolic syndrome: a multifaceted risk factor for kidney stones. Scandinavian journal of urology. 2014;48(5):414-9.

28. A.Hesse ,H.Schneider ,S.Schroder et al. Ergebnisse der AIV-gerechten Auswertung von 10000 Harnsteinanalysenbelegen. Zschr Uro Bd. 1976;69:1-9.

29. B.Shekarriz,HF Lu,ML Stoller. Correlation of unilateral urolithiasis with sleep posture. The Journal of urology. 2001;165(4):1085-7. 